\title{
RADIO EMISSION FROM HIGH REDSHIFT GALAXIES: VLA OBSERVATIONS OF THE HUBBLE DEEP FIELD
}

\author{
E.A. RICHARDS \\ University of Virginia $B$ NRAO
}

\section{Introduction}

To study galaxy populations and their evolution at the highest possible redshifts, a small area of the sky, the Hubble Deep Field (HDF) was imaged to an unprecedented sensitivity of $\mathrm{R}=29.5$ (Williams et al. 1996). As a complement to the HST observations, we have used the VLA at $8 \mathrm{GHz}$ to image an area 5!4 in diameter (FWHM) centered on the HDF to an $r m s$ sensitivity of $2 \mu \mathrm{Jy}$. With a radio resolution of about $3^{\prime \prime}$, we have 33 sources above $9.5 \mu \mathrm{Jy}$, seven in the $4 \operatorname{arcmin}^{2}$ HDF field of which six have clear optical IDs. There are an additional 12 IDs in the HST flanking fields. The optical counterparts of the radio sources are a mixture of ellipticals, spirals, and irregulars, consistent with earlier surveys of comparable depth (Windhorst et al. 1995). With a median redshift $\langle z\rangle \sim 1$, the radio galaxies we are sampling are somewhat more distant than the classical starbursting galaxies which dominate less sensitive radio surveys. Our HDF identifications are predominately with post-starburst galaxies, moderate power AGN, and blue irregulars (Fomalont et al. 1996).

\section{Primeval Galaxies or Nascent AGN?}

Six faint radio sources in our survey are identified with high redshift galaxy candidates $(z \geq 3)$ as evidenced by their optical spectral energy distributions (SED) (Steidel et al. 1996). Two of these radio emitters have confirmed redshifts of $z=2.845$ and $z=3.158$ (Steidel et al.). (One of the former has a measured $\mathrm{J}, \mathrm{H}$, and $\mathrm{K}$ flux (Cowie et al. 1996), and a seven band photometric $z=2.358 \pm 0.007$.) Thus we may be uncovering a new population of high redshift radio sources. At redshifts $z=2.3-3.5$, and 


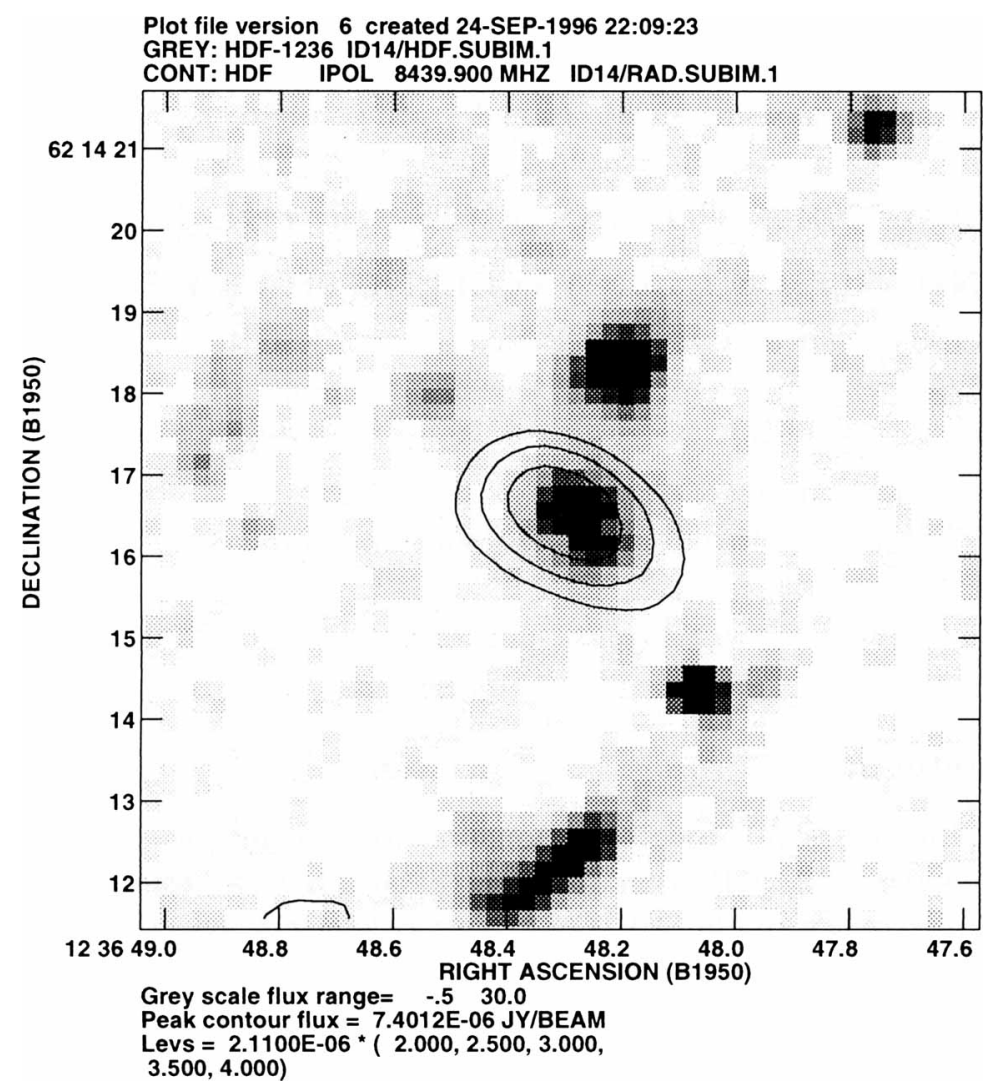

Figure 1. A J $=21.6$ galaxy (Cowie et al.): the radio contours show the unresolved $\left(\theta<3^{\prime \prime}\right)$ radio source at $8 \mathrm{GHz}$. With a $z=2.845$, the radio power is $\log \mathrm{P}=24.3$ implying either a very high star formation rate or a powerful AGN. The radio/optical coincidence is better than $0.2^{\prime \prime}$ and the identification confidence is $>99 \%$.

assuming a typical radio spectral index of -0.8 , the power of these sources at $1 \mathrm{GHz}$ is $10^{25.5} \mathrm{~W} / \mathrm{Hz}$, typical of strong FRI radio sources. Two different mechanisms may be responsible for this intense radio emission. The sources may be massive, star-forming galaxies, perhaps akin to the local ultraluminous IRAS galaxies. On the other hand, these galaxies may be relatively normal galaxies with an embedded AGN "monster."

\section{References}

Cowie, L. L. et al. 1996, Astron.J. submitted

Fomalont, E. B., et al. 1996, Astrophys.J.Lett. in press

Steidel, E. et al. 1996, Astrophys.J.Lett. 462, 17

Williams, R. et al. 1996, Astron.J. in press

Windhorst, R. A., et al. 1995, Nature 375, 471 